\title{
Lenguaje y rendimiento escolar: Un estudio predictivo
}

\author{
Grace Shum, Angeles Conde, Carmen Díaz, \\ Francisco Martínez y Lorenzo Molina
}

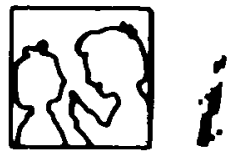

La incidencia de las competencias lingüisticas del escolar sobre las adquisiciones escolares en las demás áreas del curriculum es una realidad que todos los maestros pueden comprobar dia a día. En este trabajo se investiga esa incidencia, medida a través de la variable fracaso escolar, $y$ sus resultados podrían permitir extraer algunos principios de intervención educativa precoz en algunos aspectos del lenguaje cruciales para la escolaridad posterior.

\section{INTRODUCCION}

Desde el principio de siglo, sobre todo con la rápida tansformación tecnológica y social, la enseñanza escolar ha tomado un papel cada día más relevante. Cros (1961) afirma que el desarrollo tecnológico conlleva importantes transformaciones socio-culturales, que implica la necesidad de la enseñanza. De tal modo que dicha necesidad llega a ser uno de los elementos esenciales para la humanidad. Así, y consecuentemente, el tema del rendimiento escolar es muy candente en el campo psico-socio-pedagógico. Debido a la alta incidencia de este problema en la población escolar, se han realizado numerosas investigaciones, que desde diferentes puntos de vista tratan de descubrir y explicar las posibles causas del problema.

Los factores más estudiados al respecto son los que se refieren al desarrollo intelectual (Binet, 1911; Burt, 1921; Claparède, 1972; Foucault, 1933; Zazzo, 1945. Para estos autores, el éxito del niño en la enseñanza escolar está determinado fundamentalmente por el nivel del desarrollo de la inteligencia, sobre todo la inteligencia lógica, alcanzado por el niño. Sin embargo, Fraser (1959), Kemp (1955), etc., afirman que los aspectos motivacionales (las expectativas de los padres frente a la educación escolar, la relación que existe entre maestro y niño, et.), contribuyen al éxito o fracaso escolar. Por otro lado, algunos autores como, Hoyat (1956), Busemann 
(1939), señalan la importancia de los factores socieconómicos, relaciones familiares, hábitat, etc., en el rendimiento escolar. Por último, Bernstein (1961), Müller (1932), etc., indican que el lenguaje desempeña un papel esencial en el aprendizaje escolar.

Así, en consecuencia, el fracaso escolar es un problema complejo en el que confluyen múltiples variables. Sin embargo, entendemos que sería importante encontrar algún elemento que permita detectar en un período escolar temprano un futuro fracaso. Probablemente ese elemento sea el lenguaje, ya que éste juega un papel fundamental en el desarrollo intelectual, actuando como organizador del conocimiento y la experiencia infantil, Vygotski (1979). Desde esta perspectiva podemos considerar el lenguaje como sintetizador o indicador de la evolución del niño. Así, un niño que no haya consolidado su lenguaje materno va a encontrar problemas en su adaptación social posterior. Si añadimos a esto el hecho de que la escuela es fundamentalmente simbólica, ya que el modo de transmitir los conocimientos es eminentemente lingüístico, supone un hándicap en la adaptación del niño al medio escolar. Es decir, si el nivel de desarrollo del lenguaje natural no se halla consolidado, aparecería déficit del apendizaje en el lenguaje formal. Además, si el niño tuviese déficit en el desarrollo del lenguaje, no podría alcanzar un nivel comunicativo adecuado, así, su rendimiento y motivación se verían afectados muy negativamente. Consecuentemente, el poder determinar el grado y forma en que los diferentes aspectos del lenguaje (comprensivo y expresivo) influyen en los distintos niveles del rendimiento en la escuela, facilitaría la labor didáctica, y así se podrían elaborar planes de intervención específicos para paliar este problema.

El objetivo es intentar establecer el nivel de incidencia del lenguaje en el rendimiento escolar. Es decir, partimos de que la adquisición del lenguaje formal, portador del conocimiento y vehículo de transmisión de éste, tiene su origen en el lenguaje natural. Así, por tanto, la consolidación del lenguaje natural es determinante en el rendimiento escolar. Si pudiésemos encontrar aquellos aspectos del lenguaje que afectan y se relacionan de forma directa con las actividades académicas, estaríamos en condiciones de elaborar planes de intervención para intentar sanear tales déficits. En resumen, se trata de encontrar variables que posibiliten hacer predicciones acerca del rendimiento escolar.

\section{METODO}

\section{Sujetos}

Para el presente estudio se utilizó una muestra de sujetos pertenecientes al primer ciclo de EGB. Se emplearon 13 colegios públicos situados en distintas zonas de Madrid. Inicialmente, en el primer curso, utilizamos una muestra de 600 niños. Sin embargo, al final del estudio, la muestra se quedó reducida a 180 niños, distribuidos en 6 colegios de la siguiente forma: colegio 1: 60 niños; colegio 2: 39 niños; colegio 3: 7 niños; colegio $4: 7$ niños; colegio 5: 45 niños y colegio 6: 22 niños. 


\section{1}

\section{Procedimiento}

En primer lugar, se recogieron las informaciones sobre las aptitudes psicolingüísticas de los niños. Para ello, se aplicó una prueba estandarizada, ITPA, en el primer curso de EGB, ya que esta prueba cumple las condiciones necesarias para el estudio. Esto es, se adecúa a la edad de los niños estudiados, está disponible en lengua castellana, es una prueba muy utilizada en el ámbito escolar del país y, sobre todo, abarca todos aquellos aspectos que están relacionados con el lenguaje, especificándolos en las diversas áreas. De esta forma nos permite observar mejor el nivel de consolidación en las distintas áreas que están relacionadas con el lenguaje posibilitando así la intervención en aquellas áreas deficitarias y que afectan al rendimiento escolar. Las variables del ITPA que fueron utilizadas para nuestro estudio son: comprensión auditiva (ICA), comprensión visual (ICV), asociación auditiva (IAA), asociación visual (IAV), expresión verbal (IEV), expresión motora (IEM), integración gramatical (IIG), integración visual (IIV), memoria auditiva (IMA), memoria visual (IMV).

En el comienzo del segundo curso de EGB, para observar el nivel de producción lingüística, se aplicó una prueba sobre la expresión verbal del niño. A través de esta prueba intentamos observar el nivel del desarollo de la expresión lingüística infantil, ya que suponemos que tras un año de escolarización el lenguaje del niño necesariamente tiene que ser más elaborado y más formal. Además, se observa que el ITPA no mide prácticamente este aspecto del desarrollo. Dicha prueba fue aplicada a cinco colegios con una muestra de 180 niños.

La prueba de la expresión libre consiste en 4 viñetas que pertenecen a la primera historieta del ITPA. Esta prueba fue aplicada a través de la consigna: «cuéntame un cuento con estos dibujos». Utilizamos el cassette para registrar la producción lingüística de los niños. Posteriormente transcribimos literalmente los cuentos. Por último, procedemos a la segmentación para facilitar el análisis posterior. Todo este procedimiento ha sido realizado por varios jueces, lo cual intenta garantizar una mayor objetividad. Así pues, con todo ello, obtenemos dos tipos de textos de la prueba de expresión: texto íntegro y texto selecto.

- El texto íntegro: consiste en la producción del lenguaje total que el niño expresa durante el tiempo de la realización de la prueba de expresión lingüistica libre. Es decir, la transcripción literal de esta prueba, sin ningún tipo de manipulación.

- El texto selecto: una vez que obtenemos el texto, procedemos a un proceso de segmentación del texto íntegro. Es decir, eliminamos todos aquellos elementos que no cumplen función lingüística alguna, así, por ejemplo eliminamos los tartamudeos, las interrupciones, los falsos comienzos, etc.

\section{Tratamiento de las variables}

Como medida del rendimiento escolar se utilizó la puntuación media global de las calificaciones, que otorga el colegio correspondiente, de los sujetos estudiados. Estas calificaciones vienen dadas según cinco categorías, que son: 1 . insuficiente, 2 . suficiente, 3 . bien, 4 . notable y 5 . sobresalien- 
te, en las áreas: «lenguaje», «matemáticas» y «sociales». A partir de la distribución de la puntuación media obtenida (Tabla I) se estudiaron unos puntos de corte para dividir la muestra en tres grupos: bajo, medio y alto, que representan los tres valores con los que se formuló la variable del rendimiento escolar.

\section{TABLA I}

Distribución de la Nota media obtenida a partir de las notas en Lenguaje, Matemáticas y Sociales

\begin{tabular}{lrrrrr}
\hline Grupo & Valor & Freq. & Percent. & $\begin{array}{c}\text { Percent } \\
\text { valid. }\end{array}$ & $\begin{array}{c}\text { Percent } \\
\text { acumul. }\end{array}$ \\
\hline \multirow{3}{*}{ Bajo } & 1.00 & 2 & 1.1 & 1.1 & 1.1 \\
& 1.33 & 7 & 3.9 & 3.9 & 5.0 \\
& 2.00 & 29 & 16.1 & 16.1 & 21.1 \\
\hline \multirow{3}{*}{ Medio } & 2.33 & 8 & 4.4 & 4.4 & 25.6 \\
& 2.67 & 6 & 3.3 & 3.3 & 28.9 \\
& 3.00 & 34 & 18.9 & 18.9 & 47.8 \\
& 3.33 & 6 & 3.3 & 3.3 & 51.1 \\
& 3.67 & 3 & 1.7 & 1.7 & 52.8 \\
Alto & 4.00 & 24 & 13.3 & 13.3 & 66.1 \\
& 4.33 & 8 & 4.4 & 4.4 & 70.6 \\
& 4.67 & 15 & 8.3 & 8.3 & 78.9 \\
Total & 5.00 & 38 & 21.1 & 21.1 & 100.0 \\
\hline
\end{tabular}

En el estudio y tratamiento del texto selecto de la prueba de expresión verbal libre se establecieron dos niveles de análisis:

- Nivel morfológico: análisis de la producción morfológica, es decir, el número total de palabras diferentes producidas por cada sujeto estudiado (TOTD).

- Nivel sintáctico: análisis de la producción sintáctica PROSIN a partir de su complejidad. Dicho análisis consiste en:

a) Sintagmas: todos aquellos enunciados que no contienen verbo.

b) Oraciones: todos aquellos mensajes que poseen, por lo menos, un verbo. Las oraciones se clasificaron en dos tipos:

1. Simples: son mensajes que contienen sólo un verbo. El análisis realizado en este aspecto consiste en contabilizar el número de sintagmas que aparece en cada enunciado.

2. Compuestas: son enunciados que poseen más de un verbo, y que pueden ser coordinadas y subordinadas. Aquí procedemos a analizar el número de proposiciones que contiene cada oración.

La producción sintáctica utilizada (PROSIN) resulta de la transformación de las puntuaciones obtenidas en el análisis sintáctico. El criterio de 
esta transformación es el de: a mayor complejidad más puntuación (Tabla II).

\section{TABLA II}

Categorias sintácticas analizadas y ponderaciones asignadas a cada una de ellas

\begin{tabular}{lc}
\hline \multicolumn{1}{c}{ Categoria sintáctica } & Ponderación \\
\hline Sintagmas & 1.10 \\
Oraciones Simples de 1 Sintagma & 1.20 \\
Oraciones Simples de 2 Sintagmas & 1.30 \\
Oraciones Simples de 3 Sintagmas & 1.40 \\
Oraciones Simples de 4 Sintagmas & 1.50 \\
Oraciones Simples de 5 Sintagmas & 1.60 \\
Oraciones Simples de 6 Sintagmas & 1.70 \\
Orac. Comp. 2 propos. 1 sintagma/propos. & 1.80 \\
Orac. Comp. 2 propos. 2 sintagma/propos. & 1.90 \\
Orac. Comp. 2 propos. 3 sintagma/propos. & 2.00 \\
Orac. Comp. 2 propos. 4 sintagma/propos. & 2.10 \\
Orac. Comp. 2 propos. 5 sintagma/propos. & 2.20 \\
Orac. Comp. 2 propos. 6 sintagma/propos. & 2.30 \\
Orac. Comp. 3 propos. 1 sintagma/propos. & 2.40 \\
Orac. Comp. 3 propos. 2 sintagma/propos. & 2.50 \\
Orac. Comp. 3 propos. 3 sintagma/propos. & 2.60 \\
Orac. Comp. 3 propos. 4 sintagma/propos. & 2.70 \\
Orac. Comp. 3 propos. 5 sintagma/propos. & 2.80 \\
Orac. Comp. 3 propos. 6 sintagma/propos. & 2.90 \\
Orac. Comp. 4 propos. 1 sintagma/propos. & 3.00 \\
Orac. Comp. 4 propos. 2 sintagma/propos. & 3.10 \\
Orac. Comp. 4 propos. 3 sintagma/propos. & 3.20 \\
Orac. Comp. 4 propos. 4 sintagma/propos. & 3.30 \\
Orac. Comp. 4 propos. 5 sintagma/propos. & 3.40 \\
Orac. Comp. 4 propos. 6 sintagma/propos. & 3.50 \\
Orac. Comp. 5 propos. 1 sintagma/propos. & 3.60 \\
Orac. Comp. 5 propos. 2 sintagma/propos. & 3.70 \\
Orac. Comp. 5 propos. 3 sintagma/propos. & 3.80 \\
Orac. Comp. 5 propos. 4 sintagma/propos. & 3.90 \\
Orac. Comp. 5 propos. 5 sintagma/propos. & 4.00 \\
Orac. Comp. 5 propos. 6 sintagma/propos. & 4.10 \\
\hline
\end{tabular}

Así, tras la incorporación de las dos variables citadas anteriormente, se utilizaron como variables independientes:

- La edad (ED) en meses, de los niños, tomada al comienzo del primer curso.

- Las puntuaciones directas correspondientes a las subpruebas del ITPA: ICA, ICV, IAA, IAV, IEV, IEM, IIG, IIV, IMA, IMV.

- El total de la producción morfológica (TOTD).

- El total de la producción sintáctica (PROSIN).

\section{Análisis de los resultados}

Se realizó un análisis de varianza con cada una de las variables independientes para verificar si existían diferencias significativas entre grupos "bajo», "medio» y «alto" en el rendimiento. Se observa que en casi todas 
las medias de todas las variables aumentan al pasar del grupo «bajo» al «medio" y, de éste al grupo «alto» (Tabla III).

Por el contrario, también se observa que la media de edad (ED) alta corresponde al grupo de rendimiento bajo, mientras que la media baja pertenece al grupo medio. Esto puede explicarse, quizá, por dos razones. El hecho de que el grupo alto tenga una edad media superior a la del grupo medio, se debe posiblemente, a la maduración evolutiva, ya que la edad escolar está establecida por el comienzo del curso académico y no por la edad cronológica exacta. Así, hay meses, a veces incluso hasta un año, de diferencia de edad entre los niños. Mientras que una posible explicación para el fenómeno de que el grupo de mayor edad sea el grupo de rendimiento bajo, podría ser la de que entre los niños que poseen problemas escolares se encuentren aquellos que se incorporan a la escolarización más tardíamente. Por otra parte, se constatan casos de niños que son repetidores, debido a su mal rendimiento, y que, a pesar de ello, no logran conseguir un mejor resultado. Estos casos son, precisamente, los que elevan de forma considerable la edad media del grupo de rendimiento bajo. Este fenómeno se puede observar claramente en la Tabla III.

\section{TABLA III}

Medias de los grupos BAJO, MEDIO y ALTO en cada una de las variables independientes *

\begin{tabular}{|c|c|c|c|c|c|}
\hline \multicolumn{2}{|c|}{ Variables } & $\begin{array}{c}\text { Grupo } \\
\text { bajo }\end{array}$ & $\begin{array}{l}\text { Grupo } \\
\text { medio }\end{array}$ & $\begin{array}{c}\text { Grupo } \\
\text { alto }\end{array}$ & Total \\
\hline \multicolumn{2}{|l|}{ ED } & 79.3421 & 77.8246 & 78.9765 & 78.6889 \\
\hline \multicolumn{2}{|l|}{ ICA } & 37.4737 & 41.4912 & 42.3176 & 41.0333 \\
\hline \multicolumn{2}{|l|}{ ICV } & 14.8947 & 16.3158 & 15.8824 & 15.8111 \\
\hline \multicolumn{2}{|l|}{ IAA } & 20.2105 & 22.3158 & 24.6824 & 22.9889 \\
\hline \multicolumn{2}{|l|}{ IAV } & 21.0263 & 21.7895 & 23.8235 & 22.5889 \\
\hline \multicolumn{2}{|l|}{ IEV } & 33.2368 & 39.7719 & 41.2824 & 39.1056 \\
\hline \multicolumn{2}{|l|}{ IEM } & 20.6316 & 21.6842 & 23.2706 & 22.2111 \\
\hline \multicolumn{2}{|l|}{ IIG } & 16.3684 & 19.2456 & 20.2941 & 19.1333 \\
\hline \multicolumn{2}{|l|}{ IIV } & 20.9474 & 21.4737 & 23.4118 & 22.2778 \\
\hline \multicolumn{2}{|l|}{ IMA } & 8.3421 & 9.9123 & 11.0706 & 10.1278 \\
\hline \multicolumn{2}{|l|}{ IMV } & 10.0526 & 11.3333 & 12.4588 & 11.5944 \\
\hline \multicolumn{2}{|l|}{ TOTD } & 26.6316 & 28.7018 & 30.4941 & 29.1111 \\
\hline \multicolumn{2}{|l|}{ PROSIN } & 171.3158 & 176.6491 & 183.8235 & 178.9111 \\
\hline $\begin{array}{l}\text { ED } \\
\text { ICA } \\
\text { ICV } \\
\text { IAA } \\
\text { IAV } \\
\text { IEV } \\
\text { IEM }\end{array}$ & $\begin{array}{l}: \text { Edad. } \\
: \text { Comp } \\
: \text { Comp } \\
: \text { Asocia } \\
: \text { Asoci } \\
: \text { Expre } \\
\text { : Expre. }\end{array}$ & $\begin{array}{l}\text { n Auditiva (ITPA). } \\
\text { n Verbal (ITPA). } \\
\text { Auditiva (ITPA). } \\
\text { risual (ITPA). } \\
\text { erbal (ITPA). } \\
\text { lotora (ITPA). }\end{array}$ & $\begin{array}{l}\text { IIG } \\
\text { IIV } \\
\text { IMA } \\
\text { IMV } \\
\text { TOTD } \\
\text { PROSIN }\end{array}$ & $\begin{array}{l}\text { : Integración Gran } \\
\text { : Integración Visu } \\
: \text { Memoria Auditi } \\
\text { : Memoria Visual } \\
\text { : Total de la Prod } \\
\text { : Total de la Prod }\end{array}$ & $\begin{array}{l}\text { Morfológica } \\
\text { Sintáctica. }\end{array}$ \\
\hline
\end{tabular}

Hasta aquí hemos hablado de las diferencias encontradas entre las medias de los grupos, pero no del nivel de confianza, para que tales diferencias puedan ser consideradas estadísticamente. Obsérvese en la Tabla IV que sólo las variables del ITPA, salvo la variable ICV (comprensión visual), presentan resultados que indican diferencias signicativas entre los grupos. Sin embargo, cuando analizamos las diferencias entre el grupo bajo y el gru- 
TABLA IV

Cuadro resumen de los Análisis de Varianza de cada variable respecto de los.tres grupos de rendimiento escolar

\begin{tabular}{lcccc}
\hline & Anova 3 grupos & \multicolumn{2}{c}{ Dif. medias grupos bajo-alto } \\
\hline \multicolumn{1}{c}{ Variable } & Valor F & $\begin{array}{c}\text { Prob. (tablas) } \\
\text { G.L. 2-177 }\end{array}$ & Valor T & $\begin{array}{c}\text { Prob. (tablas) } \\
\text { G.L. 177 }\end{array}$ \\
\hline EDAD & 1.8443 & 0.1612 & -0.4435 & 0.6579 \\
ICA & 4.5778 & 0.0115 & 2.9838 & 0.0032 \\
ICV & 1.3576 & 0.2599 & 1.2183 & 0.2247 \\
IAA & 13.4462 & 0.0000 & 5.0087 & 0.0000 \\
IAV & 3.3216 & 0.0384 & 2.2967 & 0.0228 \\
IEV & 9.8647 & 0.0001 & 4.3942 & 0.0000 \\
IEM & 4.4025 & 0.0136 & 2.7956 & 0.0058 \\
IIG & 9.4870 & 0.0001 & 4.3503 & 0.0000 \\
IIV & 4.6743 & 0.0105 & 2.6431 & 0.0090 \\
IMA & 10.016 & 0.0001 & 4.4509 & 0.0000 \\
IMV & 4.7810 & 0.0095 & 3.0360 & 0.0028 \\
TOTD & 1.1255 & 0.3268 & 1.4743 & 0.1422 \\
PROSIN & 2.3917 & 0.9444 & 2.0816 & 0.0388 \\
\hline
\end{tabular}

po alto, la variable PROSIN se incorpora al grupo de variables que permiten diferenciar entre ambos grupos con un nivel de significación superior al $95 \%$. Tenemos, pues, trece variables independientes y diez de ellas pueden diferenciar, con un alto nivel de probabilidad de hacerlo bien, entre los grupos de alto y bajo rendimiento.

El interés de nuestro estudio consiste en establecer elementos de lenguaje que nos sirvan para predecir el posible bajo rendimiento posterior de un niño. Asimismo, una intervención a tiempo viene facilitada por una oportuna predicción en aptitudes deficitarias o con carencias que afectan al desarrollo normal del lenguaje.

Dichos elementos del lenguaje esperamos hallarlos más nítidamente y menos contaminados comparando el grupo bajo con el alto, lo que nos llevó a no incluir el grupo medio en este estudio comparativo de grupos. No porque no tenga importancia, sino porque la prioridad está en detectar a tiempo aquella carencia o dificultad en la capacidad y dominio del lenguaje, que pueda suponer un obstáculo o una desventaja inicial para el niño que se incorpora a la escuela. De este modo, se podría prevenir el posible fracaso escolar.

No obstante, conviene señalar que la pertenencia al grupo de bajo rendimiento no es sinónimo de suspenso ni fracaso -de hecho en este grupo están incluidos los sujetos con resultado global de suficiente (Tabla II)-, significa, más bien, que se pertenece al grupo que como tal tiene más probabilidades de encontrarse problemas para su adaptación al medio escolar. Por todo lo mencionado, la significación que nos interesa no es sólo la del valor de $\mathrm{F}$ en el análisis de varianza, sino la del valor de $\mathrm{T}$, correspondiente a la diferencia de medias entre el grupo alto y el grupo bajo de rendimiento, que tiene interés especial para este estudio.

Por último, se realizó un análisis discriminante, con todas las variables utilizadas en los análisis de varianza, el cuál nos permite obtener una función de clasificación de cada uno de los casos. Aplicando dicha función po- 
drán hacerse predicciones sobre la pertenencia a uno de los tres grupos de rendimiento, a partir de las puntuaciones en las variables discriminantes.

Con respecto a los parámetros generales utilizados en el análisis discriminante, se ha asignado a la variable PROSIN el nivel 1 para ser forzada su inclusión en la función discriminante, ya que nos interesa especialmente ver su comportamiento al tratarse de una construcción propia y novedosa. De esta manera, evitamos que su comunalidad con variables de mayor peso la excluyera de la función discriminante.

Se excluye de la clasificación al grupo de rendimiento medio, por razones mencionadas anteriormente, siendo la asignación inicial a tres grupos necesaria, ya que, de este modo, nos permitió utilizar como referencia para el corte un área (grupo medio) en lugar de tener que elegir un punto para tal efecto. Elección que siendo decisiva para el análisis no resulta fácil, sobre todo, cuando la categorización de la variable es arbitraria.

En la función de clasificación (Tabla V) se pueden apreciar las variables que intervienen en la clasificación de nuevos casos, con sus correspondientes valores. Estas variables son: cuatro subpruebas del ITPA (IAA, IEV, IMA, IMV) y la producción sintáctica (PROSIN).

\section{TABLA V}

Función de Clasificación

\begin{tabular}{lcc}
\hline Variable & Grupo Bajo & Grupo Alto \\
\hline 7 IAA & 0.60531 & 0.76195 \\
9 IEV & 0.25823 & 0.32577 \\
13 IMA & 0.49183 & 0.71221 \\
14 IMV & 0.57456 & 0.69085 \\
16 PROSIN & 0.16134 & 0.16781 \\
\hline Constante & -29.86055 & -40.49037 \\
\hline
\end{tabular}

El valor de la correlación canónica calculada para las variables que intervienen en la función discriminante $(0.56899)$ puede considerarse medioalto. Los porcentajes de clasificaciones correctas de la muestra utilizada ( $\mathrm{Ta}$ bla VI) son altos. Así, hemos de destacar que el mayor porcentaje obtenido es el que corresponde a los sujetos clasificados en el grupo de rendimiento bajo. Esto es precisamente el aspecto que hemos considerado en un principio como de máximo interés, puesto que el objetivo a alcanzar es de poder determinar los posibles problemas de malos rendimientos o futuro fracaso escolar, los cuales, a su vez, pueden ser el origen de problemas más graves.

Finalmente, se puede concluir que con la nueva variable, máxime teniendo en cuenta la dificultad de análisis que se encuentra en una prueba oral de expresión libre, puede considerarse que el resultado obtenido es esperanzador. El proceso llevado a cabo para llegar a la puntuación final ha sido difícil y laborioso. Es posible que en el camino se hay an cometido errores. No obstante, estamos seguros de que esta línea de investigación que opta por la utilización de prueba de expresión libre como soporte fundamental para la obtención de los datos habrá de fructificar a pesar de los pro- 
TABLA VI

Matriz de Clasificaciones

\begin{tabular}{|c|c|c|c|}
\hline \multirow[t]{2}{*}{ Grupo } & \multirow{2}{*}{$\begin{array}{l}\text { Porcent. } \\
\text { correcto }\end{array}$} & \multicolumn{2}{|c|}{$\begin{array}{c}\text { Número de casos } \\
\text { clasificados en cada grupo }\end{array}$} \\
\hline & & Bajo & Alto \\
\hline $\begin{array}{l}\text { Bajo } \\
\text { Medio } \\
\text { Alto }\end{array}$ & $\begin{array}{r}84.2 \\
0.0 \\
75.3\end{array}$ & $\begin{array}{l}32 \\
27 \\
21\end{array}$ & $\begin{array}{r}6 \\
30 \\
64\end{array}$ \\
\hline Total & 78.0 & 80 & 100 \\
\hline
\end{tabular}

blemas con que se enfrenta. Para ello, será necesario encontrar métodos rigurosos para el tratamiento de este tipo de datos, ya que actualmente esta carencia obliga al investigador a tomar excesivas decisiones de importancia sin la certeza suficiente.

\section{CONCLUSIONES}

A través de los resultados del análisis de los datos, puede decirse, que entre las diez variables del ITPA cuatro de ellas -IAA (asociación auditiva), IEV (expresión verbal), IMA (memoria auditiva) y IMV (memoria visual) - son utilizables como medida predictiva sobre el rendimiento escolar. Este hallazgo es importante en cuanto que permite que el niño esté sometido menos tiempo a la presión del test $\mathrm{y}$, a su vez, economiza el tiempo de corrección. Según lo dicho, se podría pensar que nuestra variable de producción sintáctica (PROSIN) sería innecesaria en la predicción del rendimiento y que con las cuatro variables del ITPA sería suficiente. Hay que tener en cuenta, por una parte, que la prueba ITPA es un test que sólo mide las aptitudes psicolingüísticas -el nivel de desarrollo cognitivo infantil- y no la capacidad del lenguaje del niño propiamente dicha. La única variable del ITPA que mide la producción del lenguaje y es significativa es la expresión verbal (EV). Sin embargo, esta medida es insuficiente, ya que únicamente mide la producción de conceptos y no la expresión lingüística que implica toda la riqueza de un discurso estructurado. Dada la característica de la prueba, es precisamente PROSIN la que trata de medir esta riqueza.

Respecto a las tres variables restantes del ITPA (IAA, IMA e IMV), decir que son medidoras de habilidades del proceso comprensivo más que del proceso expresivo. Así, una buena puntuación en estas tres variables nos da una buena capacidad de comprensión del lenguaje por parte del niño, pero no el nivel de producción. Así pues, por esta misma razón, dicha prueba no contempla la verdadera expresión lingüística, sino que se centra, más bien, en el lenguaje comprensivo.

La competencia lingüística conlleva dos habilidades: comprensiva y productiva. Una buena medida predictiva del rendimiento tiene que hacerse sobre el total de la competencia y no sólo sobre un aspecto. Esto es así, porque el lenguaje es el portador de conocimientos y vehículo de comunica- 
ción y expresión. Un buen rendimiento exige una buena competencia lingüística. Del mismo modo, si el niño presenta déficit en el desarrollo del lenguaje, tendrá un bajo rendimiento escolar.

Por todo lo anteriormente mencionado, se puede decir que PROSIN no sólo contribuye a la predicción del rendimiento, sino, tras esta medida, se puede detectar el nivel del desarrollo lingüístico y los aspectos más deficitarios de éste. Por otra parte, PROSIN mide la competencia lingüística real del niño, completándose, de este modo, la medida del ITPA. Esto es, mediante las variables del ITPA podemos detectar el nivel de las distintas aptitudes del lenguaje que adquiere el niño. Mientras que a través de la variable PROSIN podemos conocer la capacidad lingüística, tanto comprensiva como expresiva, que posee el niño. Ambas medidas son distintas, sin embargo, se complementan mutuamente. Además, si utilizamos la variable PROSIN como medida predictiva, se puede suprimir la variable IEV del ITPA, ya que este item mide precisamente la aptitud expresiva del lenguaje y es el item menos elaborado del ITPA.

Después del análisis del estudio, se nos confirma que el lenguaje está íntimamente relacionado con el rendimiento escolar. A través de la variable PROSIN se puede predecir sobre el nivel del rendimiento escolar. Sin embargo, dada la complejidad en que se encuentra el propio lenguaje, es necesario profundizar el estudio. Investigando, de este modo, no sólo el nivel sintáctico, sino también en los aspectos semántico y pragmático. Si somos capaces de encontrar un método adecuado de análisis del lenguaje en estos tres niveles que nos sirva como modelo predictivo del rendimiento, nos ayudaría a obtener informaciones suficientes sobre aquellos aspectos del lenguaje más deficitarios. Así, sería posible elaborar un programa de intervención para evitar el posible fracaso escolar. Dada la escasez, de trabajos realizados al respecto, se hace difícil el estudio. No obstante, por el papel que desempeña en el campo escolar, tiene primordial importancia e interés de continuar su estudio.

\section{Referencias}

AzCoAGA, J. E. (1981). Del lenguaje al pensamiento verbal. México, El Ateneo.

BANDRÉS, M. P. y otros (1982). La influencia del entorno educativo en el niño. Madrid, Kapelusz.

BEREITER, C. y ENGELMANN, S. (1977). Enseñanza especial preescolar. Barcelona, Fontanella.

BERNSTEIN, B. (1961). Social structure, language and learning. Educational Research, 3 , 163-176.

BERNSTEIN, B. y BRANDIS, W. (1969). Social class differences in communication and control, en Brandis, W. y Henderson, D. Social class language and communication. Londres, Routledge y Kegan Paul.

BERNSTEIN, B. y HENDERSON, D. (1969). Social class differences in the relevance of language to socialization. Sociology, 3, 1-20.

BERNSTEIN, B. y YOUNG, D. (1967). Social class differences in conceptions of uses of toys. Sociology, 1, 131-140.

BINET, A. (1911). Nouvelles recherches sur la mesure du niveau intellectuel chez les enfants d'école. L'Année psycologique, 8, 145-201.

BRASLANSKY, B. P. (1983). La lectura en la escuela. Madrid, Kapelusz.

BRESSON, P. (1970). Lenguaje oral, lenguaje escrito. En Ajuriaguerra y otros. La dixlesia en cuestión. Madrid, Pablo del Río.

BOADA, H. (1986). El desarrollo de la comunicación en el niño. Barcelona, Anthropos.

BuRT, C. (1939). Mental and Scholastic tests. Londres.

BusEmANN, A. (1939). Wohnung und Schulzensuren bei Volksschulkindern. Zeit fi Kinderforschung, 39 . 
Claparede, E. (1972). Cómo diagnosticar las aptitudes en los escolares. Madrid, Aguilar.

CrySTAL, D. y otros. (1984). Análisis gramatical de los trastomos del lenguaje. Barcelona, Médica y Técnica.

Cross, L. (1961). L'explosion scolaire, París, CUIP.

Espín, J. V. (1987). Lectura, lenguaje y educación compensatoria: teorias, modelos e instrumentos para la innovación curricular. Barcelona, Oikos-tau.

FouCAult, M. (1933). La mesure de l'intelligence. Delagrave, París.

FRASER, E. (1959). Environment and the School. Londres, Univ. of London Press.

GILLY, M. (1978). El problema del rendimiento escolar. Barcelona, Oikos-tau.

Hallahan, D. P. y Kauffman, J. M. (1985). Las dificultades en el aprendizaje. Salamanca, Anaya.

HotYaT, F. (1956). Enquète sur le rendement scolaire en première année primaire. Enfance, $9, n .{ }^{\circ} 4,31-48$.

KEMP, L. C. D. (1955). Environmental and other characteristics determining attainment in primary schools. The British Journal of Educational Psychology 25, n. $2,67-77$.

LABOV, W. (1983). Modelos sociolingüisticos. Madrid, Cátedra.

LURIA, A. R. (1979). Lenguaje y desarrollo intelectual del niño. Madrid, Pablo del Río.

LURIA, A. R. (1980). Conciencia y lenguaje. Madrid, Pablo del Río.

MÜLLER, A. (1932). Abhàngigkeit der Schulieistungen von wirtschaftlichen und sozialen Einflüssen. Arch. Gs. Ps. 1/2, 119-196.

OlERON, P. (1979). El niño y la adquisición del lenguaje. Madrid, Morata.

RONDAL, J. A. (1980). Lenguaje y educación. Barcelona, Médica y Técnica.

SigUÁN, M. (1979). Lenguaje y clase social en la infancia. Madrid, Pablo del Río.

TOUGH, J. (1987). El lenguaje oral en la escuela: una guia de observación y actuación para el maestro. Madrid, Visor.

TRIADO, C. y FORNS, M. (1989). La evaluación del lenguaje: una aproximación evolutiva. Barcelona, Anthropos.

VYGOTSKI, L. S. y otros. (1973). Psicologia y pedagogía. Madrid, Akal.

VYGOTSKI, L. S. (1973). Pensamiento y lenguaje. Buenos Aires, Pléyade.

- (1979). El desarrollo de los procesos psicológicos superiores. Barcelona, Crítica.

\section{Lenguaje y rendimiento escolar: Un estudio predictivo. G. Shum, A. Conde, C. Diaz, F. Martinez y L. Molina. CLEE, 1990, S, pp. 69-79}

\section{Resumen}

El presente trabajo tiene como objetivo establecer el nivel de incidencia del lenguaje en el rendimiento escolar. Con el fin de encontrar variables que posibiliten hacer predicciones acerca del rendimiento académico. Se utilizó una muestra de 600 sujetos pertenecientes al primer ciclo de $E G B$, de los cuales se recogieron datos sobre sus aptitudes psicolingüísticas y de su producción verbal espontánea. Así como sus calificaciones escolares. Tras aplicar los correspoondientes análisis estadisticos se encontraron correlaciones positivas en varios aspectos.

Datos sobre el autor: Grace Shum, primera autora de este trabajo, es profesora de Psicología Evolutiva en la Facultad de Psicología de la Universidad Complutense. Sus trabajos de investigación sobre la adquisición y desarrollo del lenguaje, tanto de las primeras edades como de la edad escolar, han sido objeto de diversas publicaciones.

Dirección: Universidad Complutense de Madrid, Facultad de Psicologia, Departamento de Psicología Evolutiva y de la Educación, Campus de Somosaguas, 28023 Madrid.

(C) De todos los artículos. Deberá solicitarse por escrito autorización de CL\&E y de los autores para el uso en forma de facsímil, fotocopia o cualquier otro medio de reproducción impresa. CL\&E se reserva el derecho de interponer las acciones legales necesarias en aquellos casos en que se contravenga la ley de derechos de autor. 